\title{
First-Principles Calculations for Structural, Elastic, Electronic and Thermodynamic Properties of $\mathrm{HfZn}_{2}$ under Pressure
}

\author{
GuO-Jun Li ${ }^{a}$, Lan-Ting Shi ${ }^{a}$, Cui-E Hu ${ }^{b, *}$, Yan Cheng ${ }^{a}$ And GuAng-Fu Ji ${ }^{c}$ \\ ${ }^{a}$ College of Physical Science and Technology, Sichuan University, Chengdu 610065, China \\ ${ }^{b}$ College of Physics and Electronic Engineering, Chongqing Normal University, Chongqing 400047, China \\ ${ }^{c}$ National Key Laboratory for Shock Wave and Detonation Physics Research, Institute of Fluid Physics, \\ Chinese Academy of Engineering Physics, Mianyang 621900, China \\ (Received November 22, 2017; in final form February 28, 2018)

\begin{abstract}
By using density functional theory within the Perdew-Burke-Ernzerhof generalized gradient approximation implemented in the VASP code, we study the structural, elastic, electronic, and thermodynamic properties of $C 15$ Laves-phase compound $\mathrm{HfZn}_{2}$. Comparing the lattice constants calculated from the Perdew-Burke-Ernzerhof generalized gradient approximation and local density approximation, we find that the former is in better agreement with the experimental data. The elastic constants of $\mathrm{HfZn}_{2}$ calculated by strain-stress method indicate that they keep stable up to $100 \mathrm{GPa}$. The bonding characteristics are discussed by analyzing the energy band structure, charge density distribution and charge density difference. Phonon dispersion curves and phonon density of states of $\mathrm{HfZn}_{2}$ at the different pressure are predicted for the first time. In addition, there is no imaginary frequency in the phonon band at different pressure, which also shows that $\mathrm{HfZn}_{2}$ is stable up to $100 \mathrm{GPa}$. Vibrational models are also illustrated based on phonon and group theory. The thermodynamic properties under high temperature and high pressure are calculated by different thermodynamic models. The heat capacity at constant pressure and low temperature calculated by quasi-harmonic approximation is more close to the measurement than that calculated by quasi-harmonic Debye models.
\end{abstract}

DOI: 10.12693/APhysPolA.133.1299

PACS/topics: $C 15$ Laves-phase, elastic properties, electronic structure, vibrational properties, quasi-harmonic approximation

\section{Introduction}

It has a great importance to study the Laves phase intermetallic compounds due to their interesting characteristics including polymorphism, peculiar magnetic, electrical properties $[1,2]$. Some of them are expected as hydrogen storage materials [3-5] and potential hightemperature structural materials [6] with excellent corrosion and oxidation resistance. Besides, the earlytransition-metal Laves-phase materials have anomalous elastic properties [7-9], which can be attributed to an electronic band-structure effect. The Laves phases formed by main group metallic elements and transition metals as well as by lanthanides and actinides can be classified into three structure type: the cubic $C 15$, hexagonal $C 14$, and hexagonal $C 36$. $\mathrm{ZrZn}_{2}$ with the Laves phases structure $(C 15)$ has widely attracted both theoretical and experimental attention [10-15], such as the low temperature thermal properties, electronic structure, and itinerant magnetism ferromagnetic superconducting. As an exchange enhanced paramagnet, $\mathrm{HfZn}_{2}$ has the same structure and electronic structure as $\mathrm{ZrZn}_{2}$, hence the investigation into the $5 d$ compound should be also quite interesting. The magnetic and low temperature thermal properties of $\mathrm{HfZn}_{2}$ have been studied in the past [1618]. In 2014, the elastic and thermodynamic properties

*corresponding author; e-mail: cuiehu@126.com of $\mathrm{HfZn}_{2}$ and $\mathrm{ZrZn}_{2}$ under high pressures were studied from first-principles calculations [19]. Atikur Rahman et al. investigated the optical properties of $\mathrm{HfZn}_{2}$ and thought that it should be a good coating material based on reflectivity being high in the visible-ultraviolet region up to $16 \mathrm{eV}$ [20]. In addition, they reported the superconductivity of $\mathrm{HfZn}_{2}$ at $T_{c} \approx 0.049 \mathrm{~K}$ by first-principles method [21].

The Debye temperature $\theta_{\mathrm{D}}$ is one of the most important parameter that determines the thermal characteristics of materials, but we find the values of the Debye temperature have a little discrepancy in different experiments conducted $[16,17]$. The only calculated Debye temperature based on quasi-harmonic Debye model [19] is just in accordance with the result of Ikeda et al. [17]. Furthermore, the vibrational properties of $\mathrm{HfZn}_{2}$ have not ever been investigated. To obtain a comprehensive and accurate knowledge of $\mathrm{HfZn}_{2}$, we calculate the structural, elastic, electronic, vibrational, and thermodynamic properties of $\mathrm{HfZn}_{2}$ under high pressures. The Debye temperature calculated through the elastic constants method is compared with other results. In particular, the thermodynamic properties of $\mathrm{HfZn}_{2}$ under high temperatures and high pressures were investigated by more methods, such as the Debye-Einstein model, quasi-harmonic approximation (QHA). The rest of parts of this paper are organized as follows. In Sec. 2, the computation details are given. The results and discussion are shown in Sec. 3. Finally, a summary of our results is presented in Sec. 4. 


\section{Computational method}

The first-principles calculations were performed using the Vienna ab initio simulation package (VASP) [22-24] within the framework of density functional theory. The electron-ion interaction was considered in the form of the projector-augmented-wave (PAW) method [24, 25] with a plane wave cut-off energy of $550 \mathrm{eV}$, which was found to be enough for the structural, elastic, thermodynamic properties calculations, as well as electronic calculations. No significant changes was found in the key parameters when the cut-off energy was increased. For the exchange and correlation terms in the electron-electron interaction, the Perdew-Burke-Ernzerhof (PBE) [26] exchangecorrelation functionals within the generalized gradient approximation (GGA) and local density approximation (LDA) [27] were used. The hafnium $5 p^{6} 6 s^{2} 5 d^{2}$ and zinc $3 d^{10} 4 s^{2}$ orbitals were treated as valence electrons. We used the Monkhorst-Pack $K$-point mesh [28] with a grid size of $17 \times 17 \times 17$ for integration in the irreducible Brillouin zone to ensure a convergence of total energy less than $10^{-5} \mathrm{eV} /$ atom in structural and electronic calculations.

In order to obtain the vibrational properties, we employed the supercell approach to carry out the phonon calculations, where the $2 \times 2 \times 2$ supercell was adopted. Real-space force constants were calculated by using the density functional perturbation theory (DFPT) [29, 30] implemented in the VASP code. PHONOPY code [31, 32] was used for the post-processing of real-space force constants to obtain the phonon frequencies, phonon density of states, and thermodynamic properties.

\section{Results and discussion}

\subsection{Structural properties}

The lattice parameter is the essential quantity related to the structure of the material, which determines predominantly the physical properties of the material. Intermetallic compound $\mathrm{HfZn}_{2}$ belongs to the space group $F D-3 M$ (227) with cubic crystal structure. There are two formula units per unit cell in $\mathrm{HfZn}_{2}$, where $\mathrm{Hf}$ atoms sit at $8 \mathrm{a}(0,0,0)$ and $\mathrm{Zn}$ atoms occupy Wyckoff position $16 \mathrm{~d}(0.625,0.625,0.625)$. The optimized lattice constant $a_{0}$ is computed from the equilibrium volume $V_{0}$, which corresponds to the minimum energy (Fig. 1). The $E-V$ data (energy $E$ and volume $V$ ) were fitted to the thirdorder Birch-Murnaghan equation of state [33] to determine the bulk modulus $B_{0}$ and its first derivative $B^{\prime}$ at zero pressure

$$
\begin{aligned}
& E(x)=E_{0}+\frac{3}{2} B_{0} V_{0}\left[\frac{3}{2}(\xi-1) x^{\frac{3}{2}}+\frac{3}{4}(1-2 \xi) x^{\frac{4}{3}}+\right. \\
& \left.\frac{1}{2} \xi^{\frac{6}{3}}-\frac{2 \xi-3}{4}\right],
\end{aligned}
$$

where $x=V_{0} / V, \xi=\frac{3}{4}\left(B_{0}^{\prime}-4\right)$. The calculated values of the lattice parameters $a_{0}$, bulk modulus $B_{0}$ and its first derivative $B^{\prime}$ by the Perdew-Burke-Ernzerhof (LDA) and generalized gradient approximation (GGA) are listed in Table I, together with the available experimental and other theoretical results. From Table I, $a_{0}$, $B_{0}$, and $B^{\prime}$ calculated using GGA (PBE) are in reasonable agreement with the given experimental data [34] and other calculated results [19]. It is found that the results from LDA have a big difference with the other results, especially for the lattice parameter $a_{0}$, which is underestimated by $0.17 \AA$ less than the experimental value [34]. Therefore, for the other properties of $\mathrm{HfZn}_{2}$, we used GGA as the exchange-correlation term.

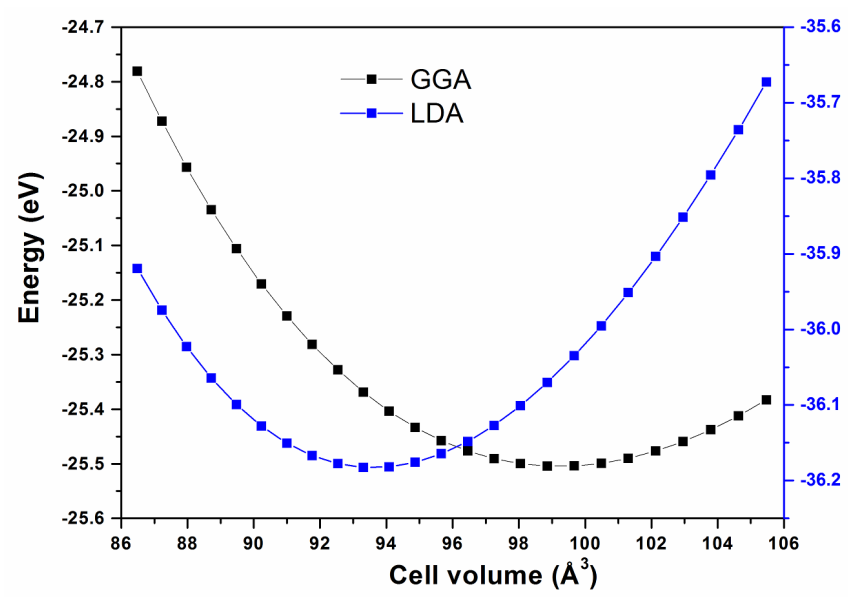

Fig. 1. Total energy versus cell volume of $\mathrm{HfZn}_{2}$ calculated using GGA (PBE) and LDA.

TABLE I

Equilibrium lattice parameters $a_{0}[\AA]$ of $\mathrm{HfZn}_{2}$ calculated using both GGA and LDA for the exchange-correlation term. The results are compared with experimental data and other results.

\begin{tabular}{c|c|c|c|c}
\hline \hline Properties & Exp. & LDA & GGA & $\begin{array}{c}\text { Other } \\
\text { calculations }\end{array}$ \\
\hline$a[\AA]$ & $7.32^{a}$ & 7.147 & 7.348 & $7.343^{b}, 7.375^{c}$ \\
$B_{0}$ & & 136.226 & 110.778 & $115.2^{b}, 160.485^{c}$ \\
$B_{0}^{\prime}$ & & 4.38 & 4.42 & $4.56^{b}$ \\
\hline${ }^{a}[34] ;{ }^{b}[19] ;{ }^{c}[20]$ &
\end{tabular}

\subsection{Elastic properties}

Mechanical and dynamical behaviors of crystals can be obtained from the elastic properties. Here, the secondorder elastic constants of $\mathrm{HfZn}_{2}$ at different pressure are calculated using strain-stress method [35]. Only three elastic constants are independent owing to the cubic structure. We find that the values of all elastic constants calculated are positive over the considered range of pressure (0-100 GPa), and satisfy the Born stability criteria $[36]$ :

$$
\begin{aligned}
& C_{11}+C_{22}+2 P>0, \quad C_{11}-C_{12}-2 P>0, \\
& C_{44}-P>0,
\end{aligned}
$$

where $P$ is external pressure. The polycrystalline bulk 
modulus $B$ and shear modulus $G$ can be obtained from the elastic constants based on the Voigt-Reuss-Hill (VRH) average scheme [37]. Here the formulae for cubic structures are

$$
\begin{aligned}
& B_{V}=B_{R}=\left(C_{11}+2 C_{12}\right) / 3, \\
& G_{V}=\left(C_{11}-C_{12}+3 C_{44}\right) / 5, \\
& G_{R}=5\left(C_{11}-C_{12}\right) C_{44} /\left[4 C_{44}+3\left(C_{11}-C_{12}\right)\right], \\
& B=\left(B_{V}+B_{R}\right) / 2, \quad G=\left(G_{V}+G_{R}\right) / 2,
\end{aligned}
$$

where Reuss and Voigt give the theoretical lower and upper bounds to the true polycrystalline $B$ and $G$, respectively. The Young modulus $E$ plays an important role in mechanical engineering design, which is estimated using the relation: $E=9 B G /(3 B+G)$.

In Fig. 2, three independent elastic constants and elastic moduli increase monotonously with pressure, and the lines of $G$ and $C_{44}$ almost close to each other owing to that the $G$ mainly results from $C_{44}$. The calculated zeropressure $B, G$ and $E$ are approximately 108.31, 56.62, and $144.65 \mathrm{GPa}$, respectively, and slightly smaller than the calculated values, $116.0,61.9$, and $157.7 \mathrm{GPa}$ for $B$, $G, E$ by Sun et al. [19].

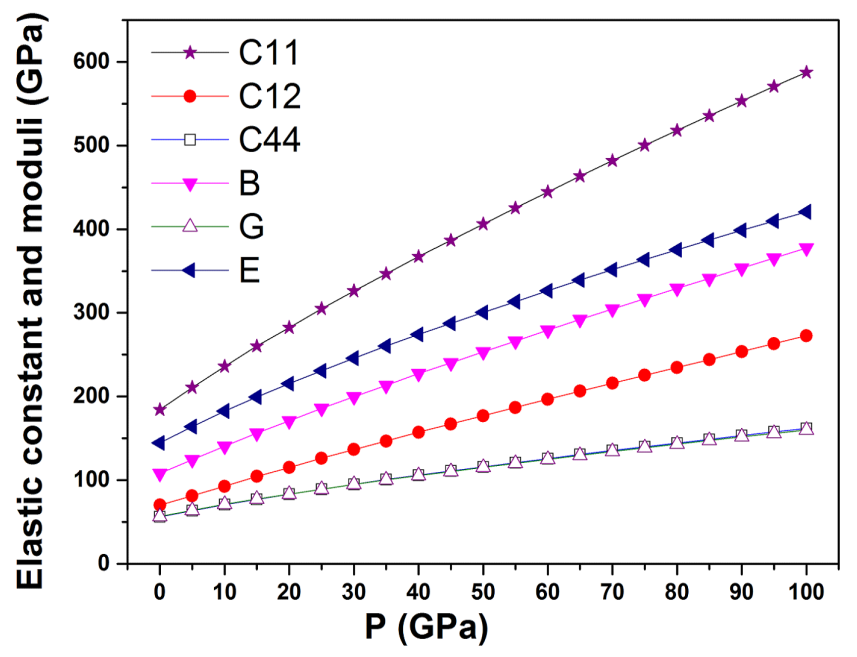

Fig. 2. The pressure dependence of elastic constant and elastic moduli calculated for $\mathrm{HfZn}_{2}$.

The Pugh criterion $(B / G)[38]$ and the Poisson ratio $\sigma$ [39] plotted in Fig. 3 are introduced to estimate the brittleness or ductility of $\mathrm{HfZn}_{2}$. If $B / G<1.75$, the material behaves in a brittle manner, otherwise, in a ductile manner. The obtained values of $(B / G)$ are always bigger than 1.75 at $0-100 \mathrm{GPa}$, indicating that $\mathrm{HfZn}_{2}$ has a good ductility. For the Poisson ratio $\sigma$, $\sigma=(3 B-2 G) /[2(3 B+G)]$, a high value always means to good ductility, whereas a low value is related to brittle nature.

The obtained values of $\sigma$, plotted in Fig. 3a, increase monotonously with pressure between $0 \mathrm{GPa}$ and $100 \mathrm{GPa}$, whose values vary from 0.277 to 0.314 . They
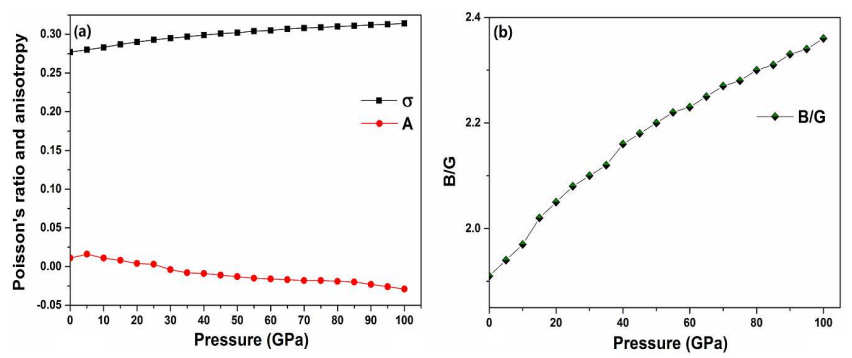

Fig. 3. (a) The pressure dependence of the Pugh ratio $B / G$ and the Poisson ratio $\sigma$, (b) anisotropy $A$ as the function of pressure.

are consistent with the $B / G$ values. Additionally, for central force solids, the Poisson ratio $\sigma=0.25$ or 0.5 are the lower limit or the upper limit, respectively [40], our value indicates that the interatomic forces in this compound are mainly central under pressure between 0 and $100 \mathrm{GPa}$. The anisotropy is calculated to get an insight of the crystal anisotropy

$$
A[41,42]=1-2 C_{44} /\left(C_{11}-C_{12}\right) .
$$

For an isotropic crystal, the value of $A$ is zero, while for an anisotropic crystal it is bigger or smaller than the zero. From Fig. 3a, we find that the values of $A$ decrease with pressure between $0 \mathrm{GPa}$ and $100 \mathrm{GPa}$ and get zero approximately at $20 \mathrm{GPa}$, suggesting that $\mathrm{HfZn}_{2}$ becomes isotropic crystal. These are a little different from the trend of $A$ by Sun et al. [19], where the values of $A$ also decrease with the increasing pressure but they are always bigger than zero. In a word, the calculated elastic constants and elastic modulus are mostly consistent with the results by Sun et al. [19] under $0 \mathrm{GPa}$ to $60 \mathrm{GPa}$, but the distinct difference still exists. This is maybe due to that the different pseudopotentials are adopted in calculations, which result in different lattice constants. Unfortunately, there are no experimental results to compare with our results.

The Debye temperature $\theta_{\mathrm{D}}$ of material at low temperature, where the vibrational excitations arise solely from acoustic vibrations, can be calculated from elastic constants using the average sound velocity $v_{m}$. The Debye temperature $\theta_{\mathrm{D}}$ is expressed as [43]:

$$
\theta_{\mathrm{D}}=\frac{\mathrm{h}}{k_{\mathrm{B}}}\left[\frac{3 n}{4 \pi}\left(\frac{N_{\mathrm{A}} \rho}{M}\right)\right]^{\frac{1}{3}} v_{m},
$$

where h, $k_{\mathrm{B}}, N_{\mathrm{A}}, n, M, \rho$ represent the Planck constant, the Boltzmann constant, the Avogadro number, the number of atoms per formula unit, the weight of molecular mass per formula unit, the density, respectively, and $v_{m}$ is obtained from

$$
v_{m}=\left[\frac{1}{3}\left(2 /\left(v_{t}\right)^{3}+1 /\left(v_{l}\right)^{3}\right)\right]^{-\frac{1}{3}} .
$$

Here, $v_{l}$ and $v_{t}$, are the longitudinal and transverse elastic wave velocities, respectively, which are obtained from the Navier equations [44]: 


$$
v_{l}=\sqrt{(3 B+4 G) / 3 \rho}, \quad v_{t}=\sqrt{G / \rho} .
$$

The calculated elastic wave velocities, the Debye temperature and other results are listed in Table II. Our calculated Debye temperature is bigger than the theoretical results of Sun et al. [19]. However, it lies in the error region of the experimental results of Radousky et al. [16] and is just a little bigger than the result of Ikeda et al. [17]. This suggests that our results are more reliable.

TABLE II

Calculated Debye temperature $\theta_{\mathrm{D}}$, average sound velocity $v_{m}$, longitudinal $v_{l}$ and transverse $\left(v_{t}\right)$ elastic wave velocities with other results.

\begin{tabular}{c|c|c|c}
\hline \hline & Our results & Exp. & $\begin{array}{c}\text { Other } \\
\text { calculations }\end{array}$ \\
\hline$V_{m}[\mathrm{~m} / \mathrm{s}]$ & 2551.55 & & \\
$V_{l}[\mathrm{~m} / \mathrm{s}]$ & 4126.74 & & \\
$V_{t}[\mathrm{~m} / \mathrm{s}]$ & 2290.80 & & \\
$\theta_{\mathrm{D}}[\mathrm{K}]$ & 302.35 & ${ }^{a} 321( \pm 25) ;{ }^{b} 289$ & ${ }^{c} 283.4$ \\
\hline
\end{tabular}

${ }^{a}[6] ;{ }^{b}[17] ;{ }^{c}[19]$

\subsection{Electronic structure and chemical bonding}

The electronic band structure can provide some important information about electronic and optical properties of materials. The calculated band structure of $\mathrm{HfZn}_{2}$ along $\mathrm{G}-\mathrm{X}-\mathrm{W}-\mathrm{K}-\mathrm{G}-\mathrm{L}-\mathrm{W}-\mathrm{X}$ direction in the full Brillouin zone is shown in Fig. 4. The yellow horizontal solid line represents the Fermi energy level. The obtained band structure is similar to those in Refs. [18, 20], illustrating that the present calculations are quite reliable. In Fig. 4, valence and conduction bands cross the Fermi level, so $\mathrm{HfZn}_{2}$ is a metal.

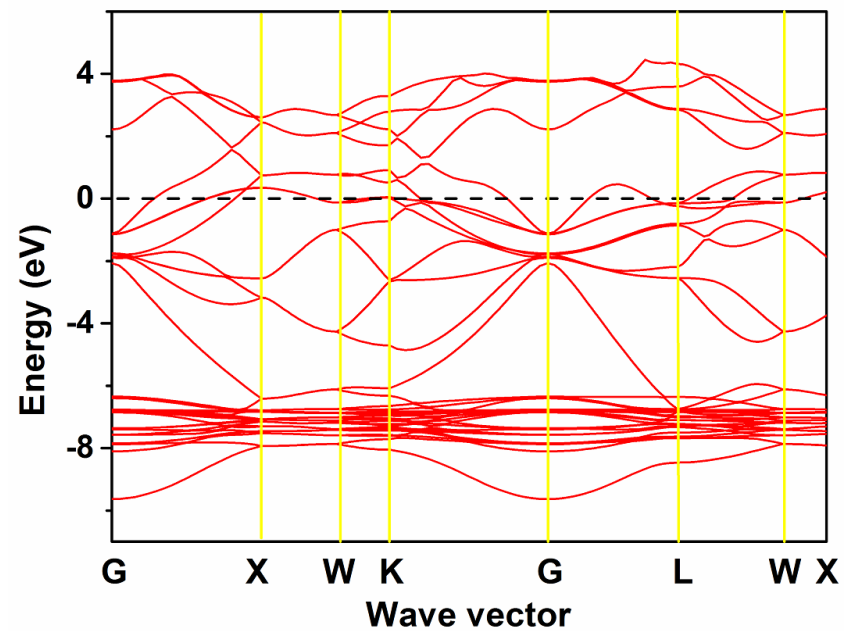

Fig. 4. Electronic band structure of $\mathrm{HfZn}_{2}$ at $0 \mathrm{GPa}$ along the high symmetry directions in the Brillouin zone.
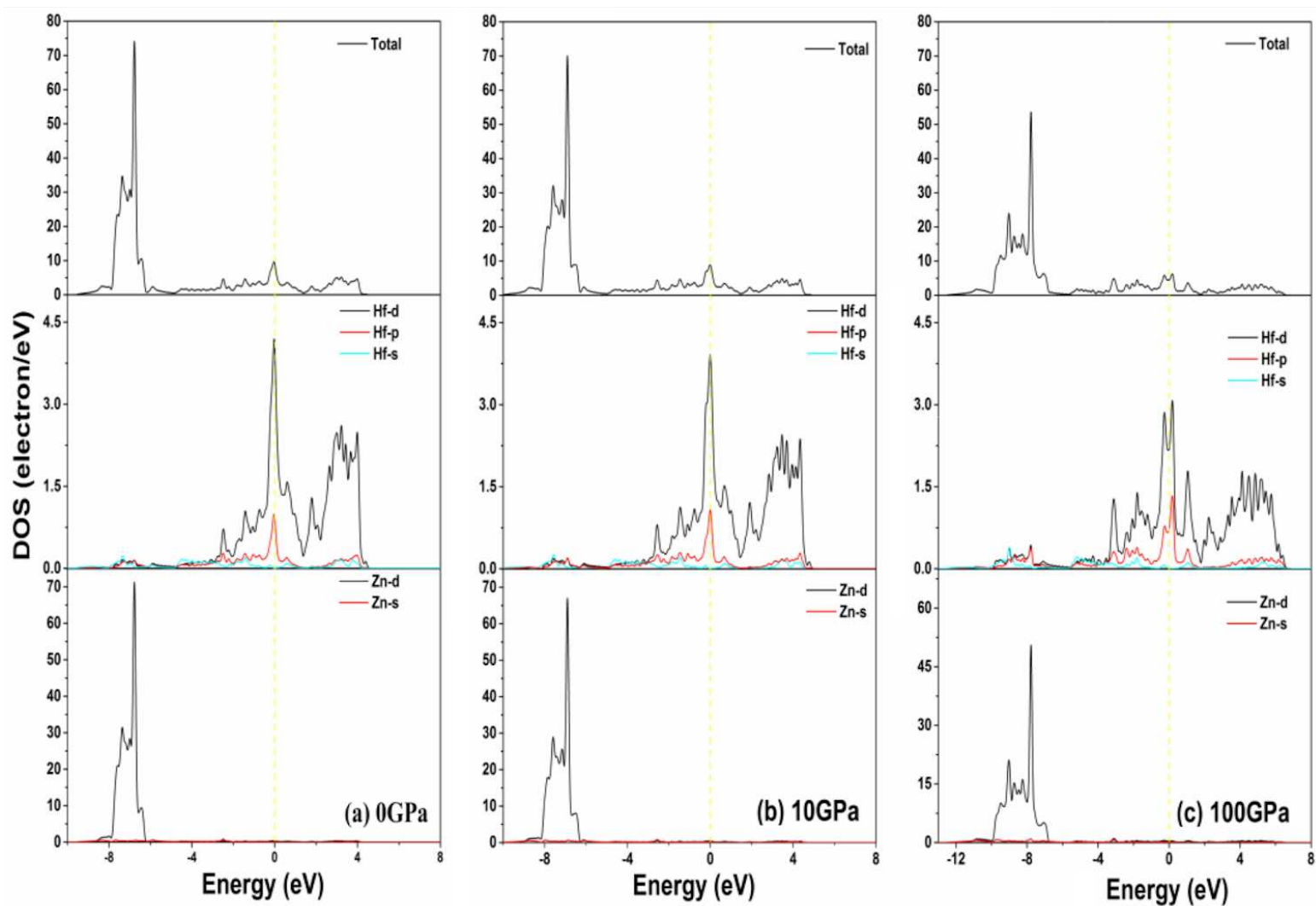

Fig. 5. Total and partial electronic density of states of $\mathrm{HfZn}_{2}$ at different pressures. The yellow dashed lines represent the position of the Fermi level. (a) $0 \mathrm{GPa}$, (b) $10 \mathrm{GPa}$, (c) $100 \mathrm{GPa}$. 
The total densities of states (TDOS) and their corresponding partial densities of states (PDOS) of $\mathrm{Hf}$ and $\mathrm{Zn}$ atoms at $0 \mathrm{GPa}$ are depicted in Fig. 5a. Based on the TDOS and PDOS, the lower energy band located at $-8.0 \mathrm{eV}$ to $-6.0 \mathrm{eV}$ are mainly composed of $\mathrm{Hf} 6 s$ and $\mathrm{Zn} 3 d$ states with a little contribution from Hf $5 d$, Hf $5 p$ and $\mathrm{Zn} 4 s$ states, The energy bands of $-6.0 \mathrm{eV}$ to $0 \mathrm{eV}$ are mainly made of $\mathrm{Hf} 5 d$, Hf $5 p, \mathrm{Zn} 4 s$ states. Moreover, we also present its TDOS and PDOS at $10 \mathrm{GPa}$ and $100 \mathrm{GPa}$ in Fig. 5b and c, respectively, to investigate the pressure effect on the electronic properties. As is shown, the tendency that the TDOS at the Fermi level $\left(N\left(E_{F}\right)\right)$ decrease with pressure is distinct $(9.638,8.672$, and 4.659 for pressure from 0 to $100 \mathrm{GPa}$. This phenomenon shows that the stability of $\mathrm{HfZn}_{2}$ gets stronger under pressure and there are similar conclusions in other electronic structure studies $[45,46]$, which show that low $N\left(E_{\mathrm{F}}\right)$ is associated with higher stability.

In order to elucidate the bonding characteristic of $\mathrm{HfZn}_{2}$, we present their valence charge density in Fig. 6a, where the blue and the red represent a low and high concentration of electrons, respectively. It can be clearly seen that the highest electric charge density occurs in the immediate vicinity of the nuclei, while the interstitial electric charge densities are relatively low. This means that most of the electrons are firmly bound up around the atomic nuclei and only a few valence electrons can escape from their bondage. Hence, there is no covalent bond in $\mathrm{HfZn}_{2}$. We also plot the charge density difference in Fig. $6 \mathrm{~b}$ to obtain the qualitative perspective on the extent of charge transfer accompanying the formation of $\mathrm{HfZn}_{2}$ compounds. It is based on the following equation [47]:

$$
\delta \rho=\rho\left(\mathrm{HfZn}_{2}\right)-\rho(\mathrm{Hf})-\rho(\mathrm{Zn}),
$$

where $\rho$ is the valence-electron charge density. In Fig. 6b, we find the loss of charge from the $\mathrm{Hf}$ and $\mathrm{Zn}$ cores is redistributed into the interstitial region. This suggests that the interstitial charge is well delocalized to form metallic bond.

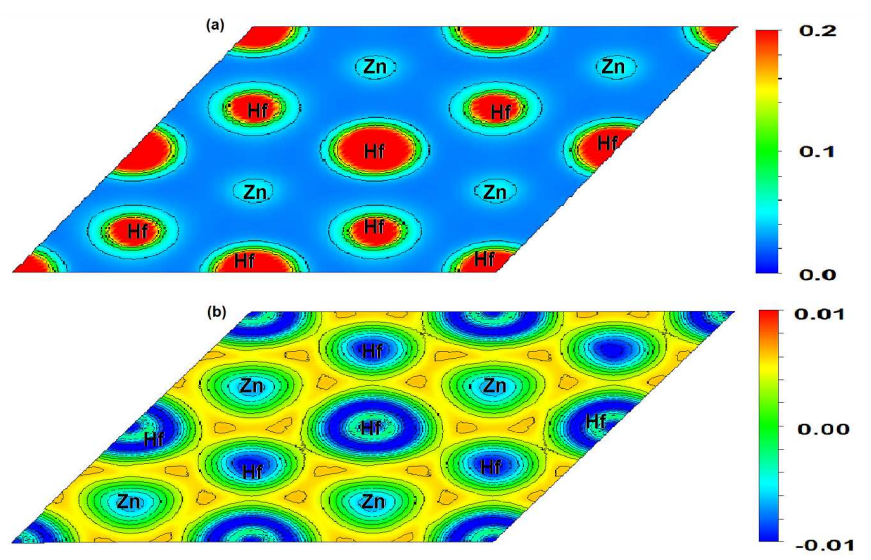

Fig. 6. The valence charge density distribution (a) and charge density difference (b) (in units of $e / \AA^{3}$ ) plot for $\mathrm{HfZn}_{2}$ computed in the (001) plane.

\subsection{Vibrational properties}

The vibrational properties play an important role in determining physical properties of solids, such as dynamical stability, phase transition, anisotropic atomic displacement parameters (ADPs). The predicted phonon spectrum along the high symmetry direction, total and partial densities of phonon states (PHDOS) of $\mathrm{HfZn}_{2}$ at 0, 10, and $100 \mathrm{GPa}$ are depicted in Fig. 7, which are
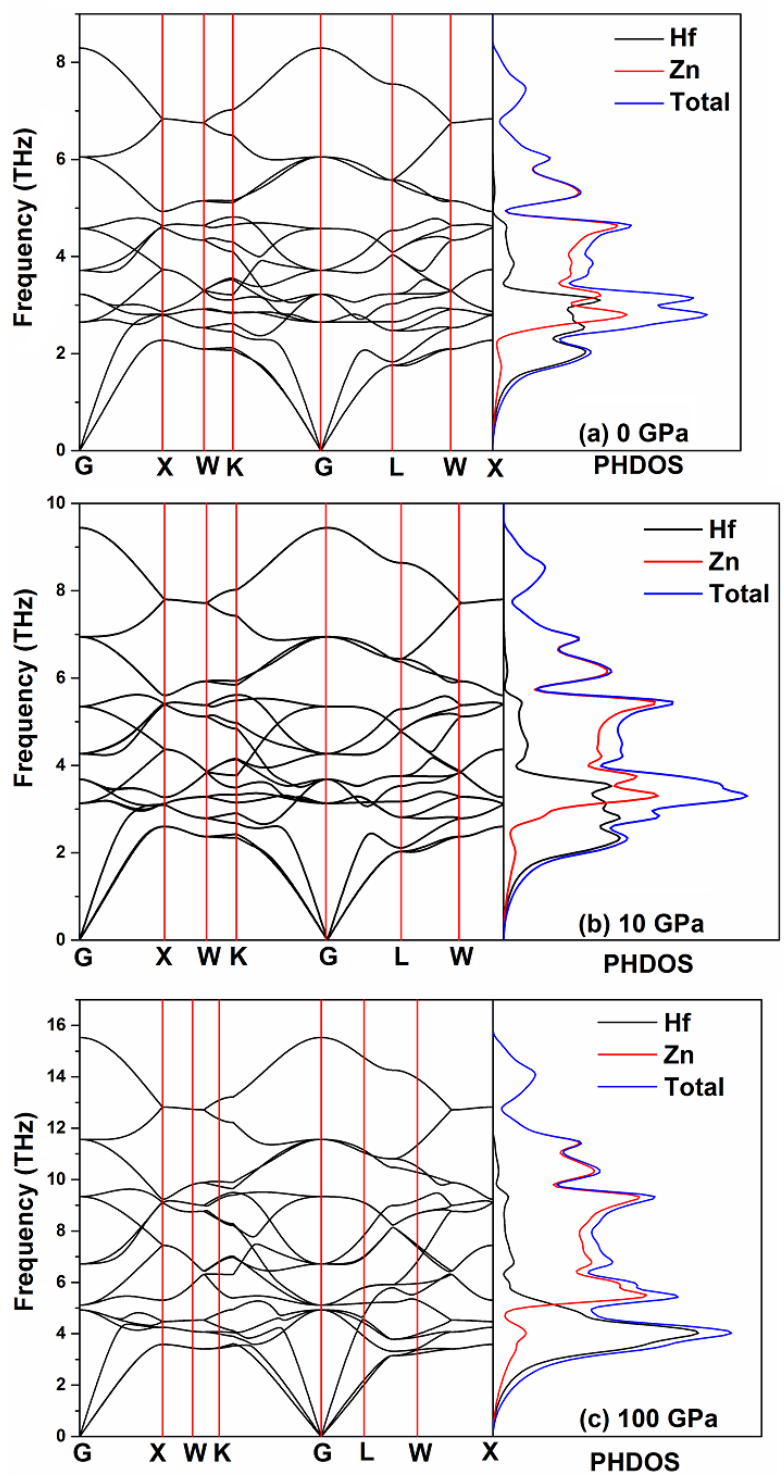

Fig. 7. Phonon dispersion curves and phonon density of states for $\mathrm{HfZn}_{2}$ compounds along several lines of high symmetry in the Brillouin zone at 0, 10, and $100 \mathrm{GPa}$, respectively.

used to examine their dynamical stability at different pressure. It is easy to find from Fig. 7 that $\mathrm{HfZn}_{2}$ is dynamically stable up to $100 \mathrm{GPa}$, since no imaginary phonon frequency is observed throughout the Brillouin zone at $100 \mathrm{GPa}$. Though there are no other theoretical calculations or experiments for the phonon of $\mathrm{HfZn}_{2}$, the stability conclusion is consistent with that from the 
elastic constant calculations. The six atoms contained in the primitive unit cell of $\mathrm{HfZn}_{2}$ can give rise to a total of 18 phonon branches, which include 15 optical modes and 3 acoustic modes. However, this number is reduced by mode degeneracies in the different directions of the Brillouin zone. All the phonon branches move to higher frequency range accordingly with the increasing pressure. At $0 \mathrm{GPa}$, the PHDOS curves of $\mathrm{HfZn}_{2}$ can be divided into two distinct regions. The low frequency region derives from hybridization between $\mathrm{Hf}$ and $\mathrm{Zn}$ atoms, but high frequency region primarily results from $\mathrm{Zn}$ atom. It is noted that the peak of PHDOS of Hf gradually gets shark with the increasing pressure, indicates that the difference of vibrational frequencies between $\mathrm{Hf}$ and $\mathrm{Zn}$ atom gets more obviously at high pressure.

Phonon modes at the Brillouin zone center are of focus in the lattice dynamics of solids. According to the irreducible representations of group theory, the optical phonon modes of $\mathrm{HfZn}_{2}$ at the Gamma point can be decomposed as

$$
\begin{aligned}
& G_{\text {optical }}=T_{2 u}(I N)+T_{1 u}(I R)+E_{u}(I N) \\
& +T_{1 u}(I R)+A_{2 u}(I R)+T_{2 g}(\mathrm{R}),
\end{aligned}
$$

where IR, R, and IN indicate the infrared, Raman and inactive silent modes, respectively. As we all known, the $T$ vibration is threefold degenerated while $E$ and $A$ vibrations are doubly and singly degenerated modes, respectively. Besides, the subscripts $u$ and $g$ represent symmetric mode and antisymmetric mode in antisymmetric center. The obtained frequencies at Gamma point are $2.650,3.2253 .717,4.576,6.054$, and 8.296 in units of THz for $T_{2 u}, T_{1 u}, E_{u}, T_{1 u}, A_{2 u}$, and $T_{2 g}$, respectively. To the best of our knowledge, there are no experimental and theoretical data available for our comparison. Referred to the vibrational analysis for cubic Laves structure $\mathrm{Al}_{2} \mathrm{Ca}$ and $\mathrm{Al}_{2} \mathrm{Mg}$ [48], similar conclusions can be obtained. $T_{2 u}, E_{u}$, and $A_{2 u}$ modes at Gamma point only include atomic vibrations of $\mathrm{Zn}$ atoms, while $T_{2 g}$ modes only include atomic vibrations of Hf atoms, but $T_{1 u}$ modes include atomic vibrations of $\mathrm{Zn}$ and Hf atoms.

\subsection{Thermodynamic properties}

After we obtained the phonon density of states (PHDOS), we investigated the thermodynamic properties of $\mathrm{HfZn}_{2}$ by means of quasi-harmonic approximation (QHA) [49] through the PHONONPY package. For QHA calculation, force constants were performed on 13 volume points by changing the optimized lattice parameter $a_{0}$. The phonon contribution to the Helmholtz energy $A(V, T)$ can be calculated by

$$
\begin{aligned}
& A(V, T)=\frac{1}{2} \sum_{\boldsymbol{q}, v} \hbar \omega_{\boldsymbol{q}, v} \\
& +k_{\mathrm{B}} T \sum_{\boldsymbol{q}, v} \ln \left(1-\exp \left(\hbar w_{\boldsymbol{q}}, v / k_{\mathrm{B}} T\right)\right),
\end{aligned}
$$

where $\boldsymbol{q}$ and $v$ are the wave vector and band index, respectively, $\omega_{\boldsymbol{q}, v}$ is the phonon frequency at $\boldsymbol{q}$ and $v, T$ is the temperature, $k_{\mathrm{B}}$ and $\hbar$ are the Boltzmann constant and the reduced Planck constant, respectively. We assume that the total Helmholtz energy,
$F(V, T)=E+A^{p h}(V, T)$, where $E$ is obtained as total energy of electronic structure at constant volume, and neglect contributions from electronic excitation, anharmonic vibrations, and other temperature-dependent effects. Then, the Gibbs free energy $G(T, P)$ at given temperature $T$ and pressure $P$ can be calculated from the Helmholtz free energy $F(T, V)$ through the following formula: $G(T, P)=\min _{V}(F(T, V)+P V)$. Using data $F(T, V)-V$, we fit them to the Vinet equation of state (EOS) and obtained related thermodynamic parameters, such as the Gibbs free energy $G$, volumetric thermal expansion coefficient $\alpha$, heat capacity at constant pressure $C_{p}$ and bulk modulus $B$.

In order to compare our results from QHA with the calculated values through the Debye-Slater mode by Sun et al. [19], we also conducted the calculation of quasiharmonic Debye modes $[50,51]$ for $\mathrm{HfZn}_{2}$, such as the Debye-Slater mode, the Debye-Grüneisen mode and the Debye-Einstein model. The details of the different quasi harmonic Debye mode calculation are not discussed here.

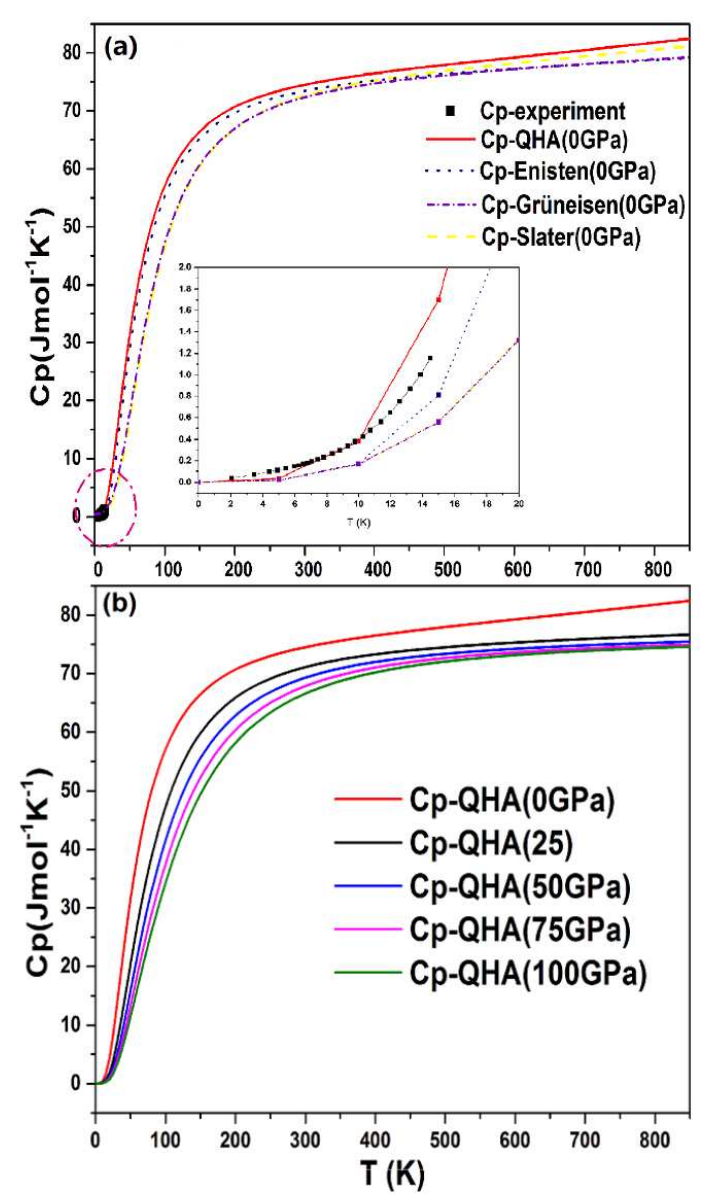

Fig. 8. (a) Temperature dependences heat capacity of $\mathrm{HfZn}_{2}$ at zero pressure from calculation and experiment [16], (b) temperature dependences heat capacity of $\mathrm{HfZn}_{2}$ at different pressure from QHA calculation. The inset in (a) is a magnified section of zero-pressure heat capacity of $\mathrm{HfZn}_{2}$ in the temperature range from 0 to $15 \mathrm{~K}$. 
In Fig. 8a, we find that the heat capacity at constant pressure $\left(C_{p}\right)$ from QHA matches with the experimental results quite well at low temperatures. This also shows that QHA is a successful theoretical method in the prediction of thermodynamic properties. The $C_{p}$ based on the Debye-Einstein model can also give a satisfying result. However, the $C_{p}$ calculated from the Debye-Slater and the Debye-Grüneisen models have a bigger discrepancy with the experimental results at $10 \mathrm{~K}$ to $15 \mathrm{~K}$. As a whole, the obtained $C_{p}$ through the Debye-Einstein model is close to the result of QHA, except in the high temperature region. Figure $8 \mathrm{~b}$ shows that the $C_{p}$ under different pressure increases very rapidly and proportionally to $T^{3}$ with the elevated temperature below $200 \mathrm{~K}$, which obeys the Debye law. When the temperature is above
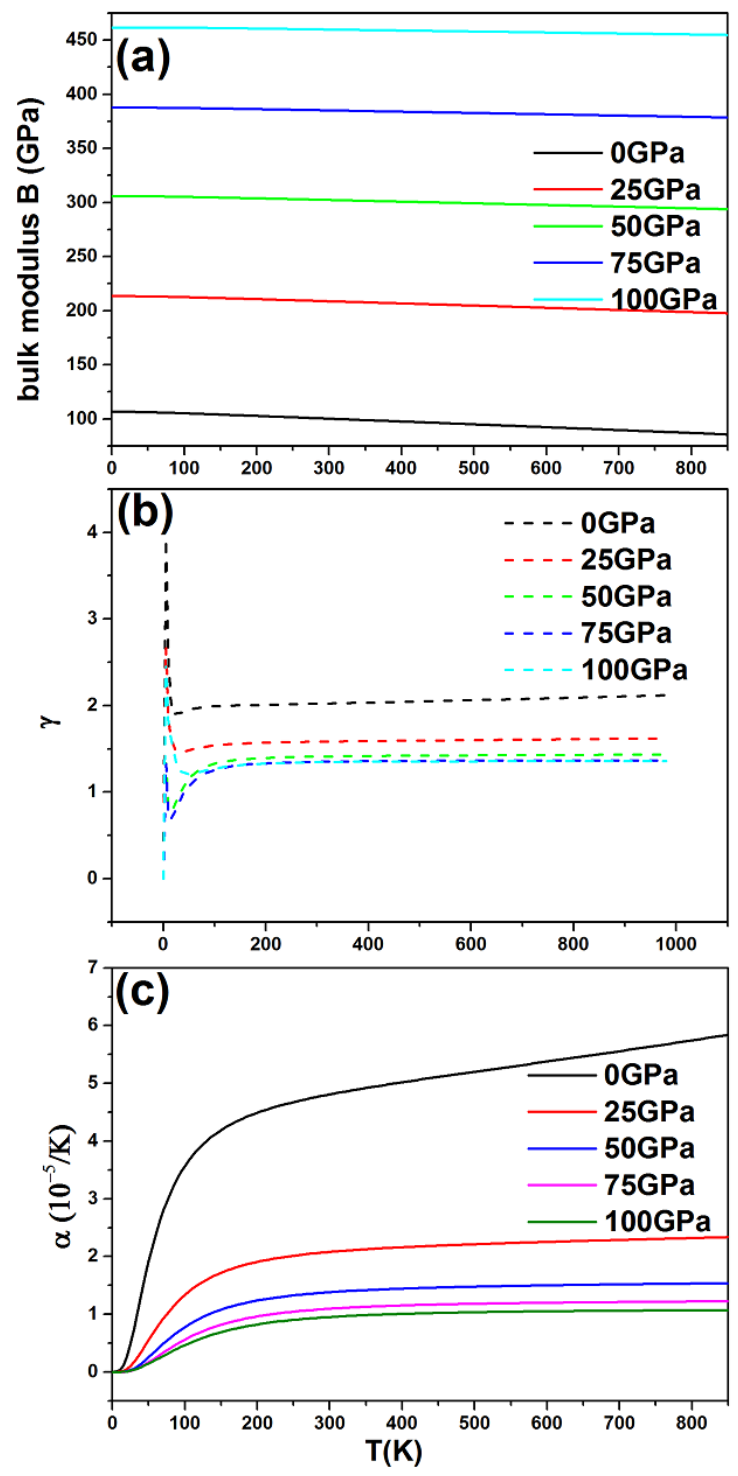

Fig. 9. Temperature and pressure dependence of bulk modulus $B$, the Grüneisen parameter $\gamma$, and thermal expansion $\alpha$ are represented in (a), (b), and (c), respectively.
$500 \mathrm{~K}$, the $C_{p}$ ascends slowly approaching approximately the Dulong-Petit limit with the temperature changing, where the influence of pressure gets smaller. Since the QHA calculation is more reliable, other thermodynamic quantities obtained by QHA are only shown.

The bulk module $B$ at $0 \mathrm{~K}$ and $0 \mathrm{GPa}$ from the QHA is 106.51 $\mathrm{GPa}$ just little smaller than the value $110.78 \mathrm{GPa}$ from the previous fitting and 108.31 GPa from the elastic constant calculations. All bulk moduli $B$ at the different pressures decrease slightly with the temperature ascending in Fig. 9a. Figure 9b shows that the temperature has prominent influence on the Grüneisen parameter $\gamma$ in the low temperature region, but the influence of pressure on $\gamma$ can be ignored in the high pressure region. The thermal expansion coefficient $\alpha$ is depicted in Fig. 9c. It is obvious that $\alpha$ is insensitive to high temperature and high pressure, and the value of $\alpha$ at 0 pressure is much greater than those at other pressures.

\section{Conclusions}

We have calculated the structural, elastic, electronic, vibration properties and thermodynamic properties for $\mathrm{HfZn}_{2}$ compounds by first-principles. Our results for equilibrium lattice parameters, bulk modulus and band structure are consistent with the previous calculated and experimental results. We show that $\mathrm{HfZn}_{2}$ is mechanically stable up to $100 \mathrm{GPa}$ according to the elastic stability criteria and phonon calculation. The obtained Debye temperature based on elastic constant method matches with the measurement quite well. Valence charge density and difference charge density indicate that $\mathrm{HfZn}_{2}$ compound is mainly made up of metallic bond. Phonon dispersion of $\mathrm{HfZn}_{2}$ is calculated at different pressures. Through QHA, the temperature and pressure dependences of heat capacity at constant pressure, bulk modulus, and thermal expansion coefficient have been discussed and compared with the results adopted quasiharmonic Debye modes.

\section{Acknowledgments}

This study was supported by the NSAF Joint built up by the National Nature Science Foundation of China and the Chinese Academy of Engineering Physics (grant No. U1430117). Some calculations were performed on the ScGrid of Supercomputing Center, Computer Network Information Center of Chinese Academy of Sciences.

\section{References}

[1] X.Q. Chen, W. Wolf, R. Podloucky, P. Rogl, M. Marsman, Phys. Rev. B 72, 054440 (2005).

[2] Y. Kishimoto, T. Ohno, Z. Naturforsch. 57a, 513 (2002).

[3] A.V. Skripov, T.J. Udovic, J.J. Rush, Phys. Rev. B 76, 104305 (2007).

[4] P. Nordlander, J.K. Norskov, F. Besenbacher, J. Phys. F Met. Phys. 16, 1161 (1986).

[5] S.L. Hong, C.L. Fu, Phys. Rev. B 66, 094109 (2002).

[6] A. Von Keitz, G. Sauthoff, Intermetallics 10, 497 (2002). 
[7] Yu.A. Izyumov, V.Ye. Naysh, V.N. Syromyatnikov, Fiz. Met. Metalloved. 39, 455 (1975).

[8] F. Chu, M. Lei, A. Migliori, S.P. Chen, T.E. Mitchell, Philos. Mag. B 70, 867 (1994).

[9] K. Foster, J.E. Hightower, R.G. Leisuret, A.V. Skripov, Philos. Mag. B 80, 1667 (2000).

[10] C. Pfleiderer, M. Uhlarz, S.M. Hayden, R. Volmer, H.V. Löhneysen, N.R. Bernhoeft, G.G. Lonzarich, Nature 412, 58 (2001).

[11] T. Smith, J. Mydosh, E. Wohlfarth, Phys. Rev. Lett. 27, 1732 (1971).

[12] G. Santi, S. Dugdale, T. Jarlborg, Phys. Rev. Lett. 87, 247004 (2001)

[13] S. Yates, G. Santi, S. Hayden, P. Meeson, S. Dugdale, Phys. Rev. Lett. 90, 057003 (2003).

[14] M. Uhlarz, C. Pfleiderer, S. Hayden, Phys. Rev. Lett. 93, 256404 (2004).

[15] E.A. Yelland, S.M. Hayden, S.J.C. Yates, C. Pfleiderer, M. Uhlarz, R. Vollmer, H.V. Löhneysen, N.R. Bernhoeft, R.P. Smith, S.S. Saxena, N. Kimura, Phys. Rev. B 72, 214523 (2005).

[16] H.B. Radousky, G.S. Knapp, J.W. Doweny, A.T. Aldred, J. Magn. Magn. Mater. 40, 117 (1983).

[17] K. Ikeda, M. Yoshizawa, K. Kai, T. Nomoto, Physica B Condens. Matter 165, 203 (1990).

[18] T. Jeong, Solid State Commun. 138, 265 (2006).

[19] N. Sun, X. Zhang, J. Qin, J. Ning, S. Zhang, S. Liang, M. Ma, R. Liu, J. Appl. Phys. 115, 083514 (2014).

[20] Atikur Rahman, Afjalur Rahman, Z. Rahaman, J. Adv. Phys. 5, 354 (2016).

[21] Z. Rahaman, Atikur Rahman, J. Adv. Phys. 6, 294 (2017).

[22] G. Kresse, J. Hafner, Phys. Rev. B 47, 558 (1993).

[23] G. Kresse, J. Furthmauller, Phys. Rev. B 54, 11169 (1996).

[24] G. Kresse, D. Joubert, Phys. Rev. B 59, 1758 (1999).

[25] P.E. Blöchl, Phys. Rev. B 50, 17953 (1994).

[26] J.P. Perdew, K. Burke, M. Ernzerhof, Phys. Rev. Lett. 77, 3865 (1996).

[27] J.P. Perdew, A. Zunger, Phys. Rev. B 23, 5048 (1981).

[28] H.J. Monkhorst, J.D. Pack, Phys. Rev. B 13, 5188 (1976).

[29] P. Giannozzi, S. de Gironcoli, P. Pavone, S. Baroni, Phys. Rev. B 43, 7231 (1991).
[30] K. Parlinski, Z.Q. Li, Y. Kawazoe, Phys. Rev. Lett. 78, 4063 (1997).

[31] A. Togo, F. Oba, I. Tanaka, Phys. Rev. B 78, 134106 (2008).

[32] A. Togo, I. Tanaka, Scr. Mater. 108, 1 (2015).

[33] J.P. Poirier, Introduction to the Physics of the Earth's Interior, Cambridge University Press, Cambridge 2000.

[34] A. Drašner and Ž. Blažina,Z. Naturforsch. 36b, 1547 (1981).

[35] Y.L. Page, P. Saxe,Phys. Rev. B 65, 104104 (2002).

[36] M.J. Mehl, B.M. Klein, D.A. Papaconstantopoulos, in: Intermetallic Compounds: Principle and Practice. Principles, Vol. 1, Eds. J.H. Westbrook, R.L. Fleischeir, Wiley, London 1995.

[37] R. Hill, Proc. Phys. Soc. A 65, 349 (1952).

[38] S.F. Pugh,Philos. Mag. 45, 823 (1954).

[39] J. Schroers, W.L. Johnson,Phys. Rev. Lett. 93 255506 (2004).

[40] D. Santamaría-Pérez, R.S. Kumar, A.J. Dos santosGarcía, D. Errandonea, R. Chuliá-Jordán, R. SaezPuche, P. Rodríguez-Hernández, A. Muñoz,Phys. Rev. B 86, 094116 (2012).

[41] B.B. Karki, L. Stixrude, S.J. Clark, M.C. Warren, G.J. Ackland, J. Crain,Am. Mineral. 82, 51 (1997).

[42] C. Zener, Elasticity and Anelasticity of Metals, University of Chicago Press, Chicago 1948.

[43] I. Johnston, G. Keeler, R. Rollins, S. Spicklemire, Solid State Physics Simulations. The Consortium for Upper-Level Physics Software, Wiley, New York 1996.

[44] W. Koster, H. Franz, Metall. Rev. 6, 1 (1961).

[45] R. Hao, X. Zhang, J. Qin, S. Zhang, J. Ning, N. Sun, M. Ma, R. Liu, RSC Adv. 5, 36779 (2015).

[46] Y. Imai, M. Mukaida, T. Tsunoda, Intermetallics 8, 381 (2000).

[47] L.G. Hector Jr., J.F. Herbst, T.W. Capehart, J. Alloys Comp. 353, 74 (2003).

[48] E. Deligoz, K. Colakoglu, H. Ozisik, Y.O. Cifti, Comput. Mater. Sci. 68, 27 (2013)

[49] A. Togo, L. Chaput, I. Tanaka, G. Hug, Phys. Rev. B 81, 174301 (2010)

[50] A. Otero-de-la-Roza, V. Luaña, Comput. Phys. Commun. 182, 1708 (2011).

[51] A. Otero-de-la-Roza, V. Luaña, Comput. Phys. Commun. 182, 2232 (2011) 